\title{
126 ANTHROPOMETRIC CHARACTERISTICS OF SELECTED COMBAT ATHLETIC GROUPS
}

Venkteshwarlu Kankanala, Eraz A Gunen, ${ }^{1}$ Ameh S Igah² ${ }^{1}$ Department of Physical and Health Education, Ahmadu Bello University, Zaria, Nigeria; ${ }^{2}$ Department of Nutrition and Dietetics, Kaduna Polytechnic, Kaduna, Nigeria

\subsection{6/bjsm.2010.078725.126}

Physical performance efficiency and effectiveness in different sports depends to a large on the size, weight and proportion of the physique of the athlete. Several kinanthropometric studies have shown significant associations between anthropometric characteristics of judo, karate and taekwondo combat athletic groups. To achieve the purpose of the study anthropometric characteristics of 80 judo (45 males and 35 females), 90 karate (40 males and 50 females) and 60 taekwondo ( 35 males and 25 females) who have been training for atleast 3 years have been measured. The anthropometric measures include body mass, standing height, abdominal circumference, waist circumference and hip circumference. These measurements were made by following the standards of anthropometric protocols of the international working group of kinanthropometrics. Differences between the male and female subjects of each group were determined. The results revealed greater waist circumference $(92.800 .75 \mathrm{~cm})$, waist hip ratio $(0.700 .01 \mathrm{~cm})$ and conicity index (174.93 1.27) of male judo groups compared to those of male karate and taekwondo groups. However male karate groups have higher mean value for triceps (7.29 0.22 $\mathrm{mm}$ ), biceps (7.73 $0.21 \mathrm{~mm}$ ) compared with those of judo and taekwondo groups, whereas male taekwondo groups have significantly higher mean value for height $(172.911 .12 \mathrm{~cm})$ and hip circumference $(136.401 .21 \mathrm{~cm})$ compared to male judo and karate groups. Among the female combat athlete groups, karate groups have significantly higher value for triceps (15.10 0.29 $\mathrm{mm}$ ) and biceps (13.30 $0.36 \mathrm{~mm}$ ) compared to the female judo and taekwondo groups. However female taekwondo group was heavier (66.08 $1.30 \mathrm{~kg}$ ) and have higher conicity index (159.69 2.01) than the female judo and karate groups. The difference between the selected female combat athletic groups in height, body mass index, abdominal circumference, waist circumference, hip circumference and waist hip ratio were insignificant. 